\title{
Cardiac Biomarkers in Patients with Cancer: Considerations, Clinical Implications, and Future Avenues
}

\author{
Valentina Bracun ${ }^{1}$. Joseph Pierre Aboumsallem ${ }^{1}$. Peter van der Meer ${ }^{1} \cdot$ Rudolf A. de Boer $^{1}$ \\ Published online: 9 June 2020 \\ (C) The Author(s) 2020
}

\begin{abstract}
Purpose of the Review As the number of cancer survivors increases due to early screening and modern (antineoplastic) treatments, cancer treatment associated cardiotoxicity (CTAC) is becoming an increasing health burden that affects survival and quality of life among cancer survivors. Thus, clinicians need to identify adverse events early, in an effort to take suitable measures before the occurrence of permanent or irreversible cardiac dysfunction.

Recent Findings Cardiac troponin (cTn) and B-type natriuretic peptide (BNP) have been proven to detect subclinical cardiotoxicity during antineoplastic treatment. As such, these cardio-specific biomarkers could predict which patients are at risk of developing CTAC even before the start of therapy. Nevertheless, there are inconsistent data from published studies, and the recommendations regarding the use of these biomarkers and their validity are mostly based on expert consensus opinion.

Summary In this review, we summarize available literature that evaluates biomarkers of CTAC, and we encourage strategies that integrate circulating biomarkers and cardiac imaging in identifying cancer patients that are at high risk.
\end{abstract}

Keywords Biomarkers $\cdot$ Chemotherapy $\cdot$ Cardiotoxicity $\cdot$ Heart failure $\cdot$ Cardio-oncology

\section{Introduction}

Now that efforts to prevent cardiovascular disease (CVD) have improved outcomes, cancer is rising as the major cause of mortality and morbidity in high-income countries [1]. According to the World Health Organization, Europe, which represents 9\% of the global population, accounts for $23.4 \%$ of the global cancer cases and $20.3 \%$ of global cancer deaths. There are approximately 8.7 million cancer survivors in Europe, [2] and more

This article is part of the Topical Collection on Cardio-oncology

Rudolf A. de Boer

r.a.de.boer@umcg.nl

Valentina Bracun

v.bracun@umcg.nl

Joseph Pierre Aboumsallem

j.abou.msallem@umcg.nl

Peter van der Meer

p.van.der.meer@umcg.nl

1 Department of Cardiology, University Medical Center Groningen, University of Groningen, AB31, PO Box 30.001, 9700

RB Groningen, the Netherlands than 3 million new cases are predicted to occur every year [3]. The Americans come in the second place with $21 \%$ and $14.4 \%$ of the global incidence and mortality, respectively [4]. According to the American Cancer Society, around 17 million Americans have a history of cancer and the number of cancer survivors is expected to increase to more than 22.1 million in 2030 [5]. Furthermore, cancer and CVD share multiple risk factors and in fact very often coincide $[6,7]$.

In the last years, cancer management and treatments have improved. However, the use of both traditional and novel antineoplastic agents is limited by their toxic effects, often leading to CTAC. Previously, the risk of CTAC was not recognized because the life span of patients with a neoplasm was too short to make CVD a major concern. Today, the progress in early diagnosis and the advances in cancer therapies have resulted in a prolonged life expectancy and improved survivorship. Consequently, the medical community recognizes the CTAC in response to antineoplastic agents as a clinically relevant issue, which has been highlighted in several recent articles [8-11, 12•].

Since functional deteriorations only become clinically manifest in later stages, when cardiotoxicity becomes irreversible, physicians should focus on early detection of CVD when treatment or prevention may still be an option [13]. Regarding 
the diagnosis of CVD, many biomarkers have demonstrated the ability to predict cardiac dysfunction before the occurrence of clinical signs or symptoms. However, the progress in the CVD biomarker field has been slow considering the fact that a good biomarker must satisfy strict criteria, including a solid and easy use of assays, good sensitivity, specificity, and knowledge on the confounders and the context of the assay. Although for several biomarker assays, these requirements are satisfied, in the setting of CTAC, this is often less straightforward. The lack of unified clinical guidelines leaves clinicians dependent on their own judgment and generalized expert opinions.

Herein, we discuss the current and future role of circulating biomarkers in the assessment of the CTAC. This review encourages to establish recommendations and guidelines on the use of circulating biomarkers and urges further research on their contribution to the pathophysiology and mechanisms mediating the CTAC. Furthermore, we would like to stress out that the old definition of cardiotoxicity does not fit the adverse effects of newer antineoplastic treatments. The revision and new systematization of CTAC are needed to meet the requirements of clinicians working in cardio-oncology practices and to better correspond with research endpoints.

\section{Cancer, an Independent Risk Factor for CVD}

Although most focus is on CTAC, there are also direct effects of cancer on the CV system. Eighty percent of all cancer patients show significant loss in muscle strength and weight, including muscle cells of the heart [14]. More than 50 years ago, Burch et al. noticed that patients who died of cancer had smaller hearts, reduced total mass, and smaller left ventricular (LV) wall thickness [15]. Cancer cells alter metabolic pathways by inducing the catabolic state in muscle cells, which leads to cardiac cachexia [14]. More recently, Pavo et al. reported that circulating cardiac biomarkers NT-proBNP and hsTnT increased with advancing tumor stage even before starting antineoplastic treatments [16]. Increased biomarker levels were strongly related to all-cause mortality. Furthermore, it has been reported that atrial natriuretic peptide (ANP), aside from its known potent diuretic, natriuretic, and vasodilatation effects, also has several immune functions [17]. It stimulates the immune system in defense against microbes, counteracts reactive oxygen species effects, and inhibits tumor growth by inducing apoptosis and necrosis. Furthermore, it has been reported that ANP and several other cardiac hormones such as long-acting NP can inhibit several tumor cell lines. Meijers et al. went a step further and showed that failing heart serves as a secreting organ that stimulates tumor growth [18•]. Recently, Shi et al. also reported a correlation between several tumor biomarkers and heart failure, showing that tumor biomarkers have an independent prognostic value in heart failure patients [19]. This link also suggests a strong overlap and possible direct pathophysiological mechanism connecting CVD and cancer $[6,7$, 20]. Therefore, the relation between cancer and heart failure appears much stronger than initially thought.

\section{Biomarkers for the Diagnosis of CTAC}

The early diagnosis of patients who are at increased risk and with asymptomatic CTAC is required to establish adequate preventive and therapeutic strategies. This could result not only in prolonged survival but also, in improved quality of life. However, cardiac follow-up based on clinical signs and symptoms of heart failure will likely result in missing a substantial proportion of patients in the early stage of cardiac dysfunction [21]. Furthermore, with the increasing trend of new antineoplastic treatments and new combination therapies, where short- and long-term complications remain peculiar, it is extremely challenging for a clinician to promptly and adequately diagnose CTAC.

In the following section, we discuss the widely studied biomarkers: cTn and natriuretic peptides, such as BNP or its stable precursor NT-proBNP. These two families are cardiospecific, which means that these proteins have been produced or released exclusively by the heart. The actual plasma levels may nevertheless be confounded by several well-known factors such as age, sex, impaired renal function, obesity, and other co-morbid conditions, such as cardiac ischemia, pulmonary embolism, and arrhythmia. Clearly, there are numerous novel biomarkers, but none of these have made into daily care [22]. This has to do with the fact that these markers are mostly not cardio-specific. We have recently shown that novel biomarkers, such as Galectin-3 (Gal-3) and growth differentiation factor (GDF)-15 are to be considered as extra-cardiac biomarkers [23]. In other words, the majority of the production is extra-cardiac, and increased plasma levels will confer information of all organs producing such factors, including tumors (Fig. 1).

\section{Cardiac Troponins, Predictors of CTAC}

In clinical practice, cTn is routinely measured in acute myocardial infarction (MI) for diagnostic and prognostic purposes [24]. Elevated cTn levels (according to the acute coronary syndrome threshold) are commonly found in conditions that either cause direct myocardial damage, excessive myocardial strain, or myocardial ischemia based on increased $\mathrm{O}_{2}$ demand or reduced $\mathrm{O}_{2}$ supply [25]. Also, cTn levels have been shown to have predictive value for most CVDs, including hypertension and (stable) coronary artery disease (CAD) in seemingly healthy individuals [26]. Several pathophysiological mechanisms in CTAC, such as oxidative stress, cardiac inflammation, fibrosis, and plaque rupture or even thrombosis, lead to 


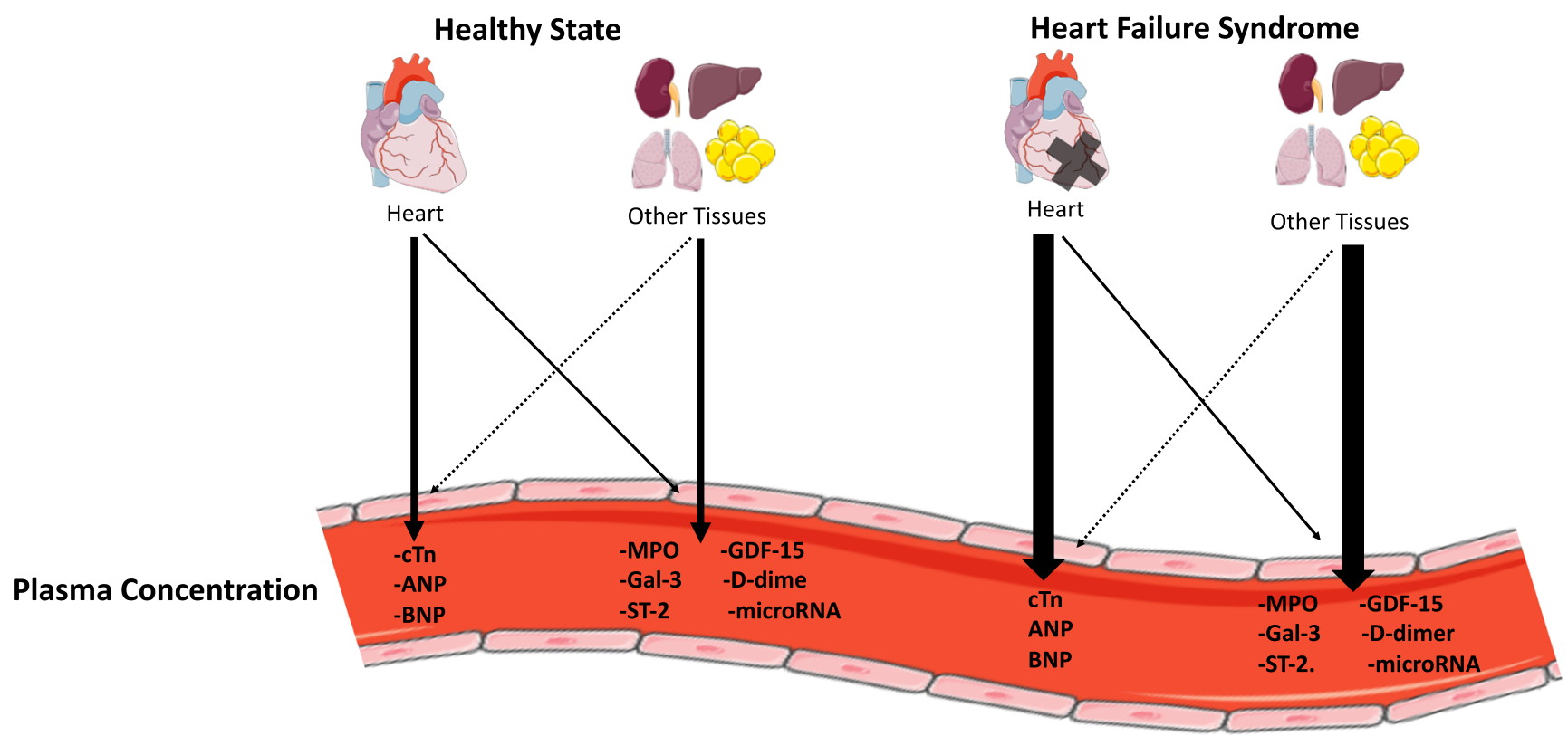

Fig. 1 Theoretical paradigm of the heart and other tissues contribution to plasma biomarkers levels. The larger arrow signifies a stronger relative contribution [23]. Illustration elements are from Smart Servier Medical Art

direct myocardial damage, and direct micro- and macrovascular impairments, which results in myocardial ischemia, and activation of the immune system (Fig. 2). Conventional and high sensitivity (hs)-cTn assays have shown prognostic values in chronically ill individuals with CVD, diabetes mellitus, chronic kidney disease, anemia, and stroke [25, 27], where elevated cTn levels have also been frequently reported $[28,29]$. Of note, such conditions are frequently observed in the cancer population, which makes it even more difficult to distinguish chemotherapy induced from classical cTn elevations [29]. cTn has been extensively studied in patients with cancer (Table 1). Ky et al. examined the possible relationship between the release of several circulating cardiac biomarkers and cardiotoxicity in patients with breast cancer receiving anthracyclines and trastuzumab [34]. The selected biomarkers were known to be involved in CTAC pathophysiological mechanisms, such as myocardial injury (cTnI), inflammation (CRP), neurohormonal activation (NT-proBNP), oxidative stress (Myeloperoxidase (MPO), and fibrosis (Gal3 ). The investigators found that elevation in absolute cTnI levels was associated with an increased risk for CTAC. This study showed that elevations in 2 or more biomarkers, in this case, $\mathrm{cTnI}$ and MPO, were associated with a higher risk of CTAC. Besides cTn and NT-proBNP, all the biomarkers used in the study were not cardio-specific, but their combination showed improved sensitivity. Blaes et al. focused their research on hs-cTn elevations in patients receiving anthracyclines [31]. They demonstrated a significant correlation between elevated baseline hs-cTn levels and the risk of developing CTAC. The authors also reported an increased risk of elevated cTn levels for asymptomatic left ventricular systolic dysfunction (LVSD) that did not meet the (strict) CTAC criteria. Their conclusion was that elevated hs-cTn levels are often observed after anthracycline treatment, not only in patients with increased CV risk but also in low-risk individuals. Their conclusion is one of great clinical relevance, showing that myocardial damage and therefore $\mathrm{cTn}$ elevations following antineoplastic treatment can be more frequently observed than initially thought. In line with this, Zardavas et al. reported an increased risk of trastuzumabrelated cardiotoxicity (TRIC) in patients with increased cTn levels after anthracycline treatment [32]. This could be explained by the increased myocardial susceptibility to damage when the combination of anthracyclines and trastuzumab is being used [45, 46]. On another hand, Kitayama et al. reported elevated absolute hs-cTn levels throughout the treatment in patients developing CTAC, while baseline hs-cTn levels did not seem to have a significant predictive value [35]. Even more compelling is the author's advocacy of a different approach to circulating biomarkers when it comes to predicting CTAC. They suggest that the integration of baseline values with hs-cTn increments could be more reliable in predicting CTAC compared with absolute hs-cTn values. The predictive value of hs-cTn is consistent among other neoplasm patients as well. Zhang et al. followed patients with B cell lymphoma receiving anthracyclines [33]. They have also reported a significant hs-cTn rise following anthracycline treatment. Those values were more prominent in patients with CTAC. Interestingly, elevated hs-cTn levels were also predictive of left ventricular diastolic dysfunction (LVDD). The authors advocated both the use of echo parameters and circulating cardiac biomarkers in predicting CTAC, seeing their 


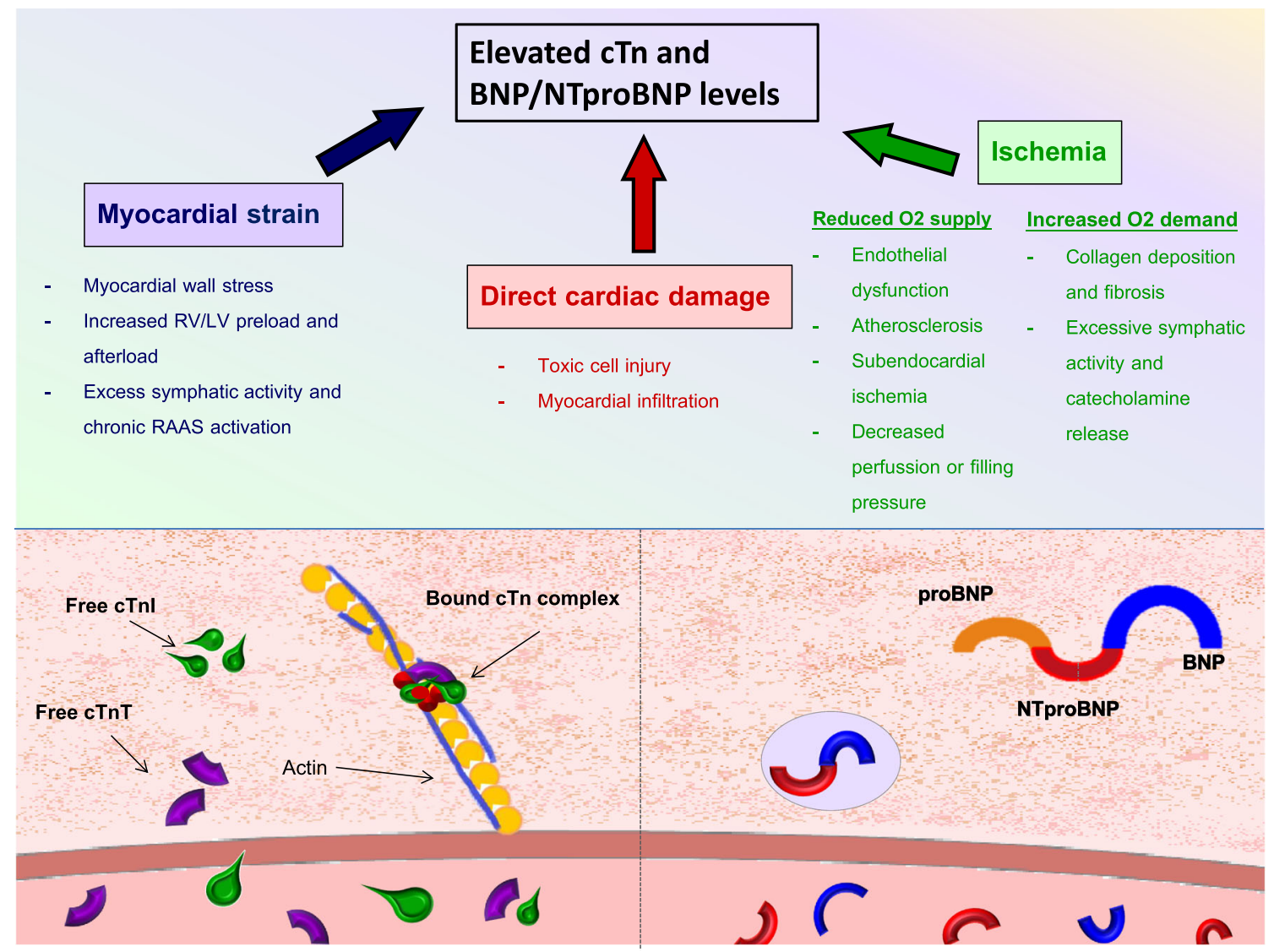

Fig. 2 The pathophysiological mechanisms of cardiotoxicity. Diverse pathophysiological mechanisms are known to cause CTAC. They are also known to cause elevated cardiac troponin (cTn) and natriuretic peptides (BNP/NTproBNP levels)

combination significantly improves sensitivity. Mahjob et al. have also emphasized the usefulness of combining circulating cardiac biomarkers with echo parameters in detecting CTAC [30]. The authors have made several important conclusions and suggested a clear correlation between elevated baseline cTn level and increased risk of CTAC, making the case for adequate $\mathrm{CV}$ risk prevention and therapy even stronger. Additionally, significant cTn elevations after the treatment with anthracyclines were also predictive of the increased CTAC risk. Demissei et al. focused their research on patients with breast cancer [43]. What makes this study so compelling is the recognition of different pathophysiological mechanisms behind anthracyclines, trastuzumab, and their combination. The authors reported that cTn elevations i.e., cardiac injury, can frequently be expected after anthracycline treatment. However, only persistently elevated cTn levels at the time of completion of anthracycline treatment, and not the baseline levels showed to be predictive of CTAC. Similarly, elevated baseline MPO levels in patients receiving anthracyclines and trastuzumab could be predictive of CTAC (HR per doubling 1.28 ; 95\% CI, 1.04-1.57, $P=0.019$ ).

Nevertheless, frequent CV risk assessment and cardiac follow-up may be favorable for the early and prompt diagnosis of CTAC for other cancer treatments, e.g., in patients receiving immune checkpoint inhibitors (ICI). Their pathophysiological mechanisms cause a wide spectrum of immune-related adverse effects including cardiac ones, (IRCAE), such as myocarditis, pericarditis, and vasculitis. IRCAE are mostly known as early adverse effects that occur in the first 90 days from the start of the treatment. Cases of fatal myocarditis are being more frequently described and it has also been suggested that its incidence is underestimated [47-49]. Mahmood et al. reported that nearly all patients (94\%) with ICI-related myocarditis had cTn elevations. In the same time, only $49 \%$ of the patients had reduced LVEF [47]. Seeing the high mortality rates of nearly $50 \%$, the baseline value and continuous $\mathrm{cTn}$ monitoring throughout the cancer treatment become even more crucial [50]. Furthermore, concerns have been raised about late IRCAE occurring 90 days after the start of ICI treatment [51]. Patients diagnosed with late IRCAE mostly show symptoms and signs of heart failure and not myocarditis. Since the different pathophysiological mechanisms determine early and late IRCAE, a different practical approach involving cardiac biomarkers (circulating and imaging) must be considered. Further research is needed to conclude the usefulness of cTn and possibly NT-proBNP in these patients. Close cardiac monitoring during, but also not any less significant, years following the cancer treatment 
Table 1 The timing of the circulating cardiac biomarker rise in response to cancer treatment. Summary of human trials dealing with cardiotoxicity and circulating cardiac biomarkers in the last 5 years

\begin{tabular}{|c|c|c|c|c|}
\hline Cancer type & Mean ANT exposure & Reported CTx $N(\%)$ & Biomarker & $\begin{array}{l}\text { Reported time of } \\
\text { significant elevation (days) }\end{array}$ \\
\hline Breast, leukemia, lymphoma [30] & $240-360 \mathrm{mg} / \mathrm{m}^{2} \mathrm{DOX}$ & $5(9.6)$ & hs-cTnI & 21 \\
\hline Breast, NHL [31] & $240-402 \mathrm{mg} / \mathrm{m}^{2} \mathrm{DOX}$ & 0 & hs-cTnT & $91-154$ \\
\hline Breast [32] & Not stated & $3(0.6)+31(7)$ & hs-cTnT, hs-cTnI & 90 \\
\hline Lymphoma [33] & $\begin{array}{l}496.2 \pm 89.4 \mathrm{mg} / \mathrm{m}^{2}-707.9 \pm 83.0 \\
\mathrm{mg} / \mathrm{m}^{2} \mathrm{DOX}\end{array}$ & $5(6)$ & hs-cTnT & $42-84$ \\
\hline Breast [34] & $240 \mathrm{mg} / \mathrm{m}^{2} \mathrm{DOX}$ & $23(24)$ & $\mathrm{cTnI}$ & 90 \\
\hline Breast [35] & $300-400 \mathrm{mg} / \mathrm{m}^{2} \mathrm{EPI}$ & $4(10)$ & hs-cTnT & 90 \\
\hline Breast [36] & $240 \mathrm{mg} / \mathrm{m}^{2} \mathrm{DOX}$ & Not stated & hs-cTnT & $2-22$ \\
\hline Breast, NHL, leukemia [37•] & $240 \mathrm{mg} / \mathrm{m}^{2} \mathrm{DOX}, 360 \mathrm{mg} / \mathrm{m}^{2}$ EPI & $3(1)$ & $\mathrm{cTn}$ & 25 \\
\hline Breast [38•] & $240-400 \mathrm{mg} / \mathrm{m}^{2} \mathrm{EPI}$ & 1 & hs-cTnI, hs-cTnT & $77-87$ \\
\hline Breast [39•] & $240 \mathrm{mg} / \mathrm{m}^{2} \mathrm{DOX}$ & $27(14)$ & hs-cTnI & 84 \\
\hline Lymphoma, sacoma, breast [40] & $308 \pm 111 \mathrm{mg} / \mathrm{m}^{2}$ (not specified) & $11(10)$ & NTproBNP & 108 \\
\hline Breast [41] & 0 & $4(9)$ & NTproBNP & $30-180$ \\
\hline Breast [42] & Not stated & $45(33)$ & NTproBNP & Not stated \\
\hline Breast [43] & $240 \mathrm{mg} / \mathrm{m}^{2}$ & $57(22 \%)$ & hs-cTnT, NT-proBNP & $0-180$ (for both) \\
\hline Lymphoma, myeloma [44] & 0 & $12 \%$ & hs-cTnT & 16 \\
\hline
\end{tabular}

$A N T$ anthracyclines, $D O X$ doxorubicine, EPI epirubicine, $C T x$ cardiotoxicity, $N H L$ non-Hodgkin lymphoma, $h s-c T n I$ high sensitive cardiac troponin I, $h s-c T n T$ high sensitive cardiac troponin $\mathrm{T}$

should be considered after ICI treatment. Another, even more recent immune therapy treatment, chimeric antigen receptor $\mathrm{T}$ cells (CAR-T) also carry significant IRCAE risks. Alvi et al. reported that up to $59 \%$ of the patients experienced cardiac damage during the CAR-T treatment, of whom $4 \%$ had cardiac-related death $[44,52]$. In their study, the investigators report that $94 \%$ of the $\mathrm{CV}$ events occurred in the patients with elevated cTn (again, according to ACS threshold). Interestingly, early treatment with tocilizumab resulted in significant reduction of $\mathrm{CV}$ events. The $\mathrm{CV}$ event risk increased by 1.7 -fold for every 12 -h treatment delay. The data validate once again the importance of IRCAE's early diagnosis, and therefore the importance of frequent and consistent measurements of circulating cardiac biomarker.

\section{cTn in Preventive and Guided Therapies}

Several other studies focused on the use of preventive and cTn-guided therapies. Gulati et al. focused their research on the possible beneficial effects of the angiotensin receptor blocker (ARB) candesartan cilexetil and the $\beta$-blocker metoprolol succinate on CTAC prevention in patients with breast cancer receiving anthracycline-based chemotherapy [38•]. The authors reported that candesartan significantly reduced the LVEF decline compared with the placebo. However, it did not affect cTn levels. Furthermore, metoprolol had no beneficial effect on the prevention of LVEF decline. A recent study from Cardinale et al. showed that despite preventive treatment with ACE inhibitors (enalapril), patients with higher anthracycline doses had elevated cTn levels throughout the treatment [37•]. Therefore, the authors supported the conclusion made by Gulati et al., which states that ARB/ACE inhibitors are less likely to prevent direct myocardial damage caused by anthracyclines (as they did not affect cTn levels), but they possibly have a beneficial effect on cardiac remodeling occurring as a result of CTAC, as they do reduce the overall CTAC incidence. Avila et al. explored the possible preventive role of another $\beta$-blocker, carvedilol in patients receiving anthracyclines [39•]. They randomized patients with breast cancer to receive carvedilol or placebo until chemotherapy completion. Their results showed that carvedilol did not affect the cardiotoxicity incidence but it reduced the surrogate outcomes, cTn levels, and LV end-diastolic diameter. At this moment, the lack of prospective data and the substantial amount of contradictory results reported in clinical trials make it impossible to draw robust conclusions on the beneficial effects of $\beta$-blockers and ARB/ACE-I in patients receiving chemotherapy. However, it is reasonable to support their use as these agents have demonstrated cardio-protective properties. Also, they are generally safe and already in use in several cardio-oncology clinics $[39,53,54]$.

\section{cTns Assays and Considerations}

One of the explanations for the broad diversity in available data may be explained by the fact that changes in conventional cTn assays, used in the first studies, were not sensitive enough to detect early, subclinical myocardial injury, as reported by 
Blaes et al. [31]. Second-generation cTns assays that measure high sensitivity (hs)-cTns, and are far more sensitive. With improved sensitivity, the possibility of optimizing the risk stratification and therefore cardiac safety, by measuring 510 times lower troponin concentrations have increased [55]. Nevertheless, several older studies reported an important role of elevated conventional cTn levels in detecting early myocardial damage due to chemotherapy [56-58].

Additional explanations could be the lack of gender and age-specific cutoff points. Recently, Rocco et al. reported that standard troponin values in younger patients and women were much lower compared with older patients and men [59]. This may be especially significant in breast cancer patients as it affects women of all ages (including the young) where high-dose protocols and combination chemotherapy treatments are used $[38,45,60]$. Moreover, it has also been postulated that sex hormones, specifically estrogen, could influence the pathophysiological mechanisms involved in anthracycline-induced cardiotoxicity [61]. Another factor that plays an important role in the heterogeneity of the reported data is the kinetics of the CTAC and timing of the blood sampling. Advani et al. showed that elevated hscTn levels could be measured within 24-48 h after the treatment with doxorubicin in breast cancer patients [36]. Elevated cTn levels were also measured after 3 weeks in all patients (11/11) treated with doxorubicin and in $7 / 11$ patients treated with trastuzumab. Other studies most frequently reported significant $\mathrm{cTn}$ increase between 42 and 154 days following the start of anthracyclines [31-35, 38, 39]. Nonetheless, different timing may be expected when it comes to ICI treatments. In most cases, IRCAE and therefore probable cTn elevations occur as early as few hours after the initial dose, up to 60 days after the initial treatment (median 30 days), as reported by Salem et al. [50]. Generally, clinical trials have set follow-up periods with scheduled echocardiography and blood sampling, with additional follow-ups if patients develop signs and symptoms of heart failure. In other words, we are considering cTn values that were quite randomly assayed, likely resulting in a lack of clear appreciation of what levels should be expected in specific stages of CTAC. Finally, a potentially important difference may be between cTnT and cTnI. cTnI is a smaller and potentially better membrane-permeable molecule [62]. It is also abundantly expressed in skeletal myopathies resulting in frequently elevated cTnI levels in patients on chronic hemodialysis [28]. A more recent analysis of hscTn assays reported that the release of hs-TnT induced by myocardial damage is smaller compared with hs-TnI [63]. As previously mentioned, conditions such as anemia, electrolyte unbalance, weight changes, and kidney dysfunction that are frequently found in the cancer population, are known to influence cTn levels and should be taken into consideration when interpreting cTn levels.
Natriuretic Peptides, Potential Predictors for CTAC

Natriuretic peptides (NPs) (e.g., BNP and NT-proBNP) are biomarkers of cardiac volume and pressure overload, mostly known for their use to diagnose acute and chronic heart failure [64]. Low BNP/NT-proBNP levels have excellent negativepredictive value, while elevated levels are indicative of the presence of heart failure. Elevated NP levels can also be found in primary pulmonary diseases and shock, atrial fibrillation, LV hypertrophy, valvular disease, and many more conditionswhere they have a strong prognostic value [27, 64-66]. Ideally, BNP/NT-proBNP results should be interpreted in consideration with age, renal function, and body mass index, as those parameters affect NP concentrations [65, 67]. While cutoff values for acute heart failure are useful to fulfill the clinical presentation and to confirm the diagnosis of heart failure, exact values in CTAC are still unknown. Therefore, for chemotherapy-induced heart failure, serial sampling might detect small but significant variations that are useful to diagnose early CTAC.

Lenihan et al. focused their research on the utility of circulating cardiac biomarkers in patients receiving anthracyclines [40]. Their findings supported the validity of BNP/NT-proBNP serial measurements in predicting anthracycline-induced CTAC. Both baseline and post-therapy BNP values, in patients that developed cardiac adverse events, were higher than in those without events. Interestingly, cTnI was not associated with worse outcomes. More recently, Bouwer et al. found a statistically significant correlation between BNP/NT-proBNP levels and trastuzumabinduced cardiotoxicity (TIC) in patients with HER2-positive breast cancer [42]. While all patients demonstrated a NTproBNP rise during the trastuzumab treatment, the difference was significantly higher in patients that developed TIC. They also reported that for every $+10 \mathrm{pmol} / \mathrm{l}$ NT-proBNP rise, LVEF declined by approximately $4 \%$. Similarly, Demissi et al. reported that the doubling of NT-proBNP in patients was associated with a $0.7 \%$ decline in LVEF at each subsequent visit [43]. Strikingly, NT-proBNP changes were different between different cancer treatment regimen groups. The changes were most evident in patients receiving anthracycline with trastuzumab, where for each NT-proBNP doubling from baseline an up to $1.3 \%$ decline in LVEF was observed. However, in patients receiving only trastuzumab, NT-proBNP levels significantly declined in the first 6 months. Zardavas et al. focused on the role of circulating cardiac biomarkers in monitoring the cardiac safety of patients with early-stage HER2-positive breast cancer receiving trastuzumab [32]. The authors reported higher BNP/ NTproBNP levels following anthracycline and before trastuzumab treatment in patients that developed TIC. Adjuvant chemotherapy treatments often come in the combination with radiotherapy. Still, only a few studies focused on the evaluation of possible cardiotoxic effects due to radiotherapy. Palumbo et al. measured BNP values in patients with left-sided breast cancer receiving adjuvant radiotherapy [41] and found 
that BNP levels were significantly elevated and rising in all patients from first, up to 6 months after the start of radiotherapy. Even 12 months after the start of radiotherapy, BNP levels remained significantly above the baseline value. At the end of the follow-up (median of 74 months), none of the patients developed heart failure and 4 patients developed (MI). While elevated BNP values did not correlate with LVEF decline, it strongly advocates the need for long-term follow-up. Furthermore, D'Errico et al. also suggested that BNP/NT-proBNP could have an important role in detecting myocardial injury and cardiac remodeling after radiation treatment in patients with left-sided breast cancer [68]. In contrast to these studies, others are showing no benefit of BNP in predicting CTAC [35, 36, 69].

These contradictory results could be explained by the heterogeneity of the cancer population and treatments used, different timing of sampling and diverse study protocols. Furthermore, the kinetics of BNP/NT-proBNP release in CTAC, just as is the case with cTn, are still unknown. Ponde et al. suggested that only patients who were treated with anthracyclines showed significant changes in circulating NTproBNP and cTn levels [69]. This indicates that for other cancer treatments, we still do not have the proper observation, prevention, and therefore no treatment options. Versatile pathophysiological mechanisms of different antineoplastic treatments and genetic predisposition to myocardial stress could result in different effects and timing of myocardial injury. Therefore, different sampling timings but also new, sex-specific cutoff points of circulating biomarkers are needed to diagnose CTAC.

\section{Other Circulating Biomarkers in Cardio-Oncology}

Several other circulating biomarkers are also being assessed for their potential use in diagnosing early myocardial injury before LV dysfunction becomes obviously impaired. As previously mentioned, biomarkers are mostly chosen because of their pathophysiological mechanisms. Researchers are focusing on biomarkers with known pathophysiological mechanisms that could potentially fit and explain processes happening during CTAC (Table 2). In addition to the already discussed circulating biomarkers of myocardial stretch and injury, biomarkers of cardiac fibrosis and oxidative stress like MPO, Gal-3, ST2, and GDF-15 are increasingly studied [34, 43, 70, 71]. Those biomarkers are, however, not cardiacspecific and aggregate research data remain inconsistent. Furthermore, several microRNAs were found overexpressed in cardiac tissue after MI predicting heart failure, while others are found overexpressed in both cardiac and tumor tissues [72].

Measuring microRNA showed some promising results in detecting early CTAC, but predominantly in animal studies. Finally, in the lack of consistent data, we strongly recommend clinicians to consider an integrated biomarker approach, combining circulating biomarkers with imaging methods. This combined technique showed indeed improved specificity, while sensitivity, as expected, dropped [9, 33, 34].

\section{Future Directions and Conclusions}

There is great relevance and need to screen or survey for CTAC with biomarkers as it may reduce the need for invasive diagnostic tools and interventions, and even costs. These biomarkers should be specific, sensitive, accurate, consistent, and reproducible [73]. Providentially, cardiac-specific circulating biomarkers have demonstrated important signals and may be useful as diagnostic and prognostic markers in the assessment of CTAC.

Table 2 List of biomarkers used in cardio-oncology studies. Summary of pathophysiological mechanisms of the most common biomarkers used in cardiology and their practical use in cardio-oncology

\begin{tabular}{llll}
\hline Biomarker & Pathophysiological mechanism & Antineoplastic treatments & Clinical presentation \\
\hline cTn (TnT, TnI) & Myocardial injury & ANT, ICI, VEGF, CAR-T, RTx & ACS, myocarditis, acute HF, arrhythmias \\
NT-proBNP & Myocardial strain & AND + HER-2 inh, TKI, PI, possible CAR-T & Acute and chronic HF \\
MPO & Oxidative stress & ANT + HER-2 inh & Unknown \\
Gal-3 & Fibrosis & ANT & Acute and chronic HF \\
ST-2 & Endothelial strain and fibrosis & ANT & Acute and chronic HF \\
GDF-15 & Ischemia, oxidative stress, strain & ANT & Acute and chronic HF, ACS \\
micro-RNA & Cell signaling & ANT & ACS, chronic heart failure \\
D-dimer & Thrombosis & TKI, VEGF-inh & DVT, PE
\end{tabular}

cTn cardiac troponins, NT proBNP natriuretic peptide, MPO myeloperoxidase, Gal-3 galactin 3, GDF-15 growth differentiation factor-15, ANT anthracyclines, ICI immune checkpoint inhibitors, TKI tyrosine kinase inhibitors, $P I$ proteasome inhibitors, $C A R-T$ chimeric antigen receptor T cells, $V E G F$-inh vascular endothelial growth factor inhibitor, $A C S$ acute coronary syndrome, $H F$ heart failure, $K F$ kidney function, $D V T$ deep venous thrombosis, $P E$ pulmonary embolism 


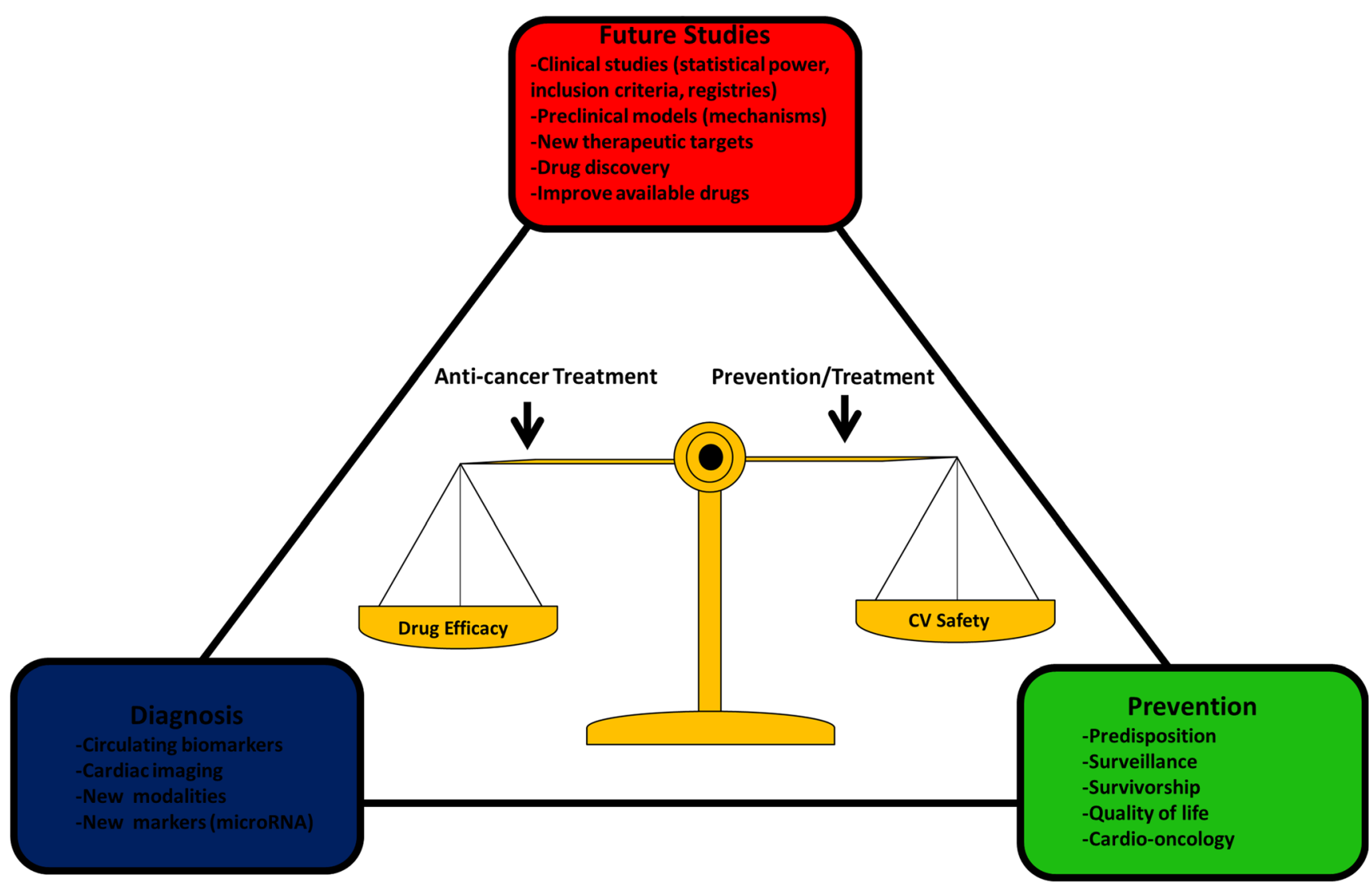

Fig. 3 Illustration of future strategies and goals to address the knowledge gap, generate accurate and reliable clinical data, improve diagnostic tools, and optimize treatment and surveillance

There are conflicting data regarding the utility of circulating biomarkers before, during, and after anti-neoplastic treatments. The inconsistencies between the published studies in this field could be avoided in the future by designing prospective trials with adequate statistical power, long-term followup, and systemic measurement of circulating biomarkers.

Furthermore, strategies that integrate circulating biomarkers and cardiac imaging have an incremental value in identifying cancer patients that are at high risk of CTAC. Also, it has been postulated that biomarker levels are age and gender-specific, which highlights the importance of precision medicine as a potential future solution. The focus should be set not only on selecting the most effective biomarkers for different cancer treatments but also on filling up the knowledge gaps, especially when it comes to the mechanism of myocardial injury. This could be done by preselecting standardized timing of the sampling, both baseline and during follow-up, which is greatly influenced by the type of cancer treatment regimen, and by detecting new circulating biomarker thresholds.

Today, most of the available data come from studies that were based on old high-dose treatment regimens and they describe treatments involving only anthracyclines, or regimens that are anthracycline-based. New treatments, such as immune therapy have shown remarkable survival outcomes. However, their long-term effects remain mostly unknown. For a clinician, the current preventive, diagnostic, and treatment options are mostly opinion-based. We would however strongly recommend routine usage of circulating cardiac biomarkers, both $\mathrm{cTn}$ and NT-proBNP, and their combination with imaging methods. This way, clinicians can recognize early signs of cardiac damage, and start treatment accordingly. cTn has also shown to have a strong negative predictive value. This may help in recognizing patients that are at low cardiac risk, and steer development of either more aggressive treatment plan, if necessary, or less strict cardiac follow-up. Further, the old definition of cardiotoxicity does not describe all known CTAC effects such as myocarditis, cardiac arrhythmias, or MI following new treatments. Adopting new definitions will most certainly increase the number of patients being diagnosed. The systematization of different cancer treatments and explanations of their pathophysiological mechanisms, leading to updated cardiotoxicity definition are also needed to adapt researchers' endpoints. This would expand our knowledge, create new clinical approaches, and is likely to improve patient care and survival. Because in the end, a safe continuation of anti-neoplastic treatment is the common goal for everyone - and especially those visiting and working in the cardio-oncology service (Fig. 3). 
Acknowledgments This work is supported by the European Research Council (ERC CoG 818715, SECRETE-HF).

\section{Compliance with Ethical Standards}

Conflict of Interest Valentina Bracun declares that she has no conflict of interest. Joseph Pierre Aboumsallem declares that he has no conflict of interest. Peter van der Meer has received consultancy fees and/or research grants from Novartis, Servier, Vifor Pharma, Astra Zenica, Pfizer, and Ionis. Rudolf A. de Boer has received speaker's honoraria from Abbott, AstraZeneca, Novartis, and Roche. Additionally, the University Medical Center Groningen, which employs Drs. de Boer and van der Meer, has received research grants and/or fees from AstraZeneca, Abbott, BristolMyers Squibb, Novartis, Novo Nordisk, and Roche.

Human and Animal Rights and Informed Consent This article does not contain any studies with human or animal subjects performed by any of the authors.

Open Access This article is licensed under a Creative Commons Attribution 4.0 International License, which permits use, sharing, adaptation, distribution and reproduction in any medium or format, as long as you give appropriate credit to the original author(s) and the source, provide a link to the Creative Commons licence, and indicate if changes were made. The images or other third party material in this article are included in the article's Creative Commons licence, unless indicated otherwise in a credit line to the material. If material is not included in the article's Creative Commons licence and your intended use is not permitted by statutory regulation or exceeds the permitted use, you will need to obtain permission directly from the copyright holder. To view a copy of this licence, visit http://creativecommons.org/licenses/by/4.0/.

\section{References}

Papers of particular interest, published recently, have been highlighted as:

- Of importance

1. Dagenais GR, Leong DP, Rangarajan S, Lanas F, Lopez-Jaramillo $\mathrm{P}$, Gupta R, et al. Variations in common diseases, hospital admissions, and deaths in middle-aged adults in 21 countries from five continents (PURE): a prospective cohort study. Lancet (London, England). 2019;19.

2. Rossi S, Baili P, Capocaccia R, Caldora M, Carrani E, Minicozzi P, et al. The EUROCARE-5 study on cancer survival in Europe 19992007: database, quality checks and statistical analysis methods. Eur J Cancer. 2015;51:2104-19.

3. Ferrell BR, Temel JS, Temin S, Alesi ER, Balboni TA, Basch EM, et al. Integration of palliative care into standard oncology care: American Society of Clinical Oncology clinical practice guideline update. J Clin Oncol. 2017;35:96-112.

4. Bray F, Ferlay J, Soerjomataram I, Siegel RL, Torre LA, Jemal A. Global cancer statistics 2018: GLOBOCAN estimates of incidence and mortality worldwide for 36 cancers in 185 countries. CA Cancer J Clin. 2018;68:394-424.

5. American Cancer Society. Facts \& Figures 2019. Am Cancer Soc: 2019.

6. Boer RA, Meijers WC, Meer P, Veldhuisen DJ. Cancer and heart disease: associations and relations. Eur J Heart Fail. 2019: ejhf.1539.
7. Meijers WC, de Boer RA. Common risk factors for heart failure and cancer. Cardiovasc Res. 2019;115:844-53.

8. Cardinale D, Biasillo G, Salvatici M, Sandri MT, Cipolla CM. Using biomarkers to predict and to prevent cardiotoxicity of cancer therapy. Expert Rev Mol Diagn Taylor \& Francis. 2017;17:245-56.

9. Curigliano G, Cardinale D, Dent S, Criscitiello C, Aseyev O, Lenihan D, et al. Cardiotoxicity of anticancer treatments: epidemiology, detection, and management. CA Cancer J Clin. 2016;66: 309-25.

10. Strongman H, Gadd S, Matthews A, Mansfield KE, Stanway S, Lyon AR, et al. Medium and long-term risks of specific cardiovascular diseases in survivors of 20 adult cancers: a population-based cohort study using multiple linked UK electronic health records databases. Lancet. The Author(s). Published by Elsevier Ltd. This is an Open Access article under the CC BY 4.0 license. 2019;394: 1041-54.

11. Zamorano JL, Lancellotti P, Rodriguez Muñoz D, Aboyans V, Asteggiano R, Galderisi M, et al. 2016 ESC Position Paper on cancer treatments and cardiovascular toxicity developed under the auspices of the ESC Committee for Practice Guidelines. Eur Heart J. 2016:37:2768-801.

12. - Michel L, Mincu RI, Mahabadi AA, Settelmeier S, Al-Rashid F, Rassaf T, et al. Troponins and brain natriuretic peptides for the prediction of cardiotoxicity in cancer patients: a meta-analysis. Eur J Heart Fail. 2019:ejhf.1631. Elaborative meta- analysis of circulating cardiac biomarkers in patients treated with anthracyclines.

13. Cardinale D, Colombo A, Bacchiani G, Tedeschi I, Meroni CA, Veglia F, et al. Early detection of anthracycline cardiotoxicity and improvement with heart failure therapy. Circulation. 2015;131: 1981-8.

14. Sweeney M, Yiu A, Lyon AR. Cardiac atrophy and heart failure in cancer. Card Fail Rev. 2017;03:62.

15. Burch GE, Phillips JH, Ansari A. The cachectic heart. Dis Chest. 1968;54:403-9.

16. Pavo N, Raderer M, Hülsmann M, Neuhold S, Adlbrecht C, Strunk $\mathrm{G}$, et al. Cardiovascular biomarkers in patients with cancer and their association with all-cause mortality. Heart. 2015;101:1874-80.

17. De Vito P. Atrial natriuretic peptide: an old hormone or a new cytokine? Peptides. 2014:108-16.

18. - Meijers WC, Maglione M, Bakker SJL, Oberhuber R, Kieneker LM, De Jong S, et al. Heart failure stimulates tumor growth by circulating factors. Circulation. 2018;138:678-91. Study showing indesputable connection between heart failure and cancer.

19. Shi C, van der Wal HH, Silljé HHW, Dokter MM, van den Berg F, Huizinga L, et al. Tumor biomarkers: association with heart failure outcomes. J Intern Med. 2020.

20. Aboumsallem JP, Moslehi J, De Boer RA. Reverse cardio-oncology: cancer development in patients with cardiovascular disease. $\mathrm{J}$ Am Heart Assoc. 2020 Jan 21;9(2):e013754.

21. Yoldaș T, Yeșil Ș, Karademir S, Șahin G, Arman Örün U, Doğan $\mathrm{V}$, et al. Evaluation of long-term cardiac side effects of anthracycline chemotherapy by conventional and nonconventional echocardiographic methods in childhood cancer survivors. Cardiol Young. 2019;29:904-9.

22. Meijers WC, De Boer RA, Van Veldhuisen DJ, Jaarsma T, Hillege HL, Maisel AS, et al. Biomarkers and low risk in heart failure. Data from COACH and TRIUMPH. Eur J Heart Fail. 2015;17:1271-82.

23. Du W, Piek A, Schouten EM, de van Kolk CWA, Mueller C, Mebazaa A, et al. Plasma levels of heart failure biomarkers are primarily a reflection of extracardiac production. Theranostics. 2018;8:4155-69.

24. Roffi M, Patrono C, Collet JP, Mueller C, Valgimigli M, Andreotti F, et al. 2015 ESC guidelines for the management of acute coronary syndromes in patients presenting without persistent ST-segment elevation. Russ J Cardiol. 2016;131:9-63. 
25. Park KC, Gaze DC, Collinson PO, Marber MS. Cardiac troponins: from myocardial infarction to chronic disease. Cardiovasc Res. 2017;113:1708-18.

26. Thygesen K, Alpert JS, Jaffe AS, Chaitman BR, Bax JJ, Morrow DA, et al. Fourth universal definition of myocardial infarction (2018). Circulation. 2018;138:e618-51.

27. Myhre PL, O’Meara E, Claggett BL, de Denus S, Jarolim P, Anand IS, et al. Cardiac troponin I and risk of cardiac events in patients with heart failure and preserved ejection fraction. Circ Heart Fail. 2018;11:e05312.

28. Tanindi A, Cemri M. Troponin elevation in conditions other than acute coronary syndromes. Vasc Health Risk Manag. 2011;7:597603.

29. Eggers KM, Jernberg T, Lindahl B. Cardiac troponin elevation in patients without a specific diagnosis. J Am Coll Cardiol. 2019;73: $1-9$.

30. Mahjoob MP, Sheikholeslami SA, Dadras M, Mansouri H, Haghi M, Naderian M, et al. Prognostic value of cardiac biomarkers assessment in combination with myocardial 2D strain echocardiography for early detection of anthracycline-related cardiac toxicity. Cardiovasc Hematol Disord Targets. 2019;19.

31. Blaes A, Rehman A, Vock D, Luo X, Menge M, Yee D, et al. Utility of high-sensitivity cardiac troponin $\mathrm{T}$ in patients receiving anthracycline chemotherapy. Vasc Health Risk Manag. 2015;11: 591.

32. Zardavas D, Suter TM, Van Veldhuisen DJ, Steinseifer J, Noe J, Lauer S, et al. Role of troponins I and $\mathrm{T}$ and $\mathrm{N}$-terminal prohormone of brain natriuretic peptide in monitoring cardiac safety of patients with early-stage human epidermal growth factor receptor 2-positive breast cancer receiving trastuzumab: a herceptin adjuvant study ca. J Clin Oncol. 2017;35:878-84.

33. Zhang CJ, Pei XL, Song FY, Guo Y, Zhang QL, Shu XH, et al. Early anthracycline-induced cardiotoxicity monitored by echocardiographic Doppler parameters combined with serum hs-cTnT. Echocardiography. 2017;34:1593-600.

34. Ky B, Putt M, Sawaya H, French B, Januzzi JL, Sebag IA, et al. Early increases in multiple biomarkers predict subsequent cardiotoxicity in patients with breast cancer treated with doxorubicin, taxanes, and trastuzumab. J Am Coll Cardiol. 2014;63:809-16.

35. Kitayama H, Kondo T, Sugiyama J, Kurimoto K, Nishino Y, Kawada M, et al. High-sensitive troponin $\mathrm{T}$ assay can predict anthracycline- and trastuzumab-induced cardiotoxicity in breast cancer patients. Breast Cancer. 2017;24:774-82.

36. Advani P, Hoyne J, Moreno-Aspita A, Dubin M, Brock S, Harlow $\mathrm{C}$, et al. High-sensitivity troponin T and NT-proBNP kinetics in breast cancer chemotherapy. Chemotherapy. 2017;62:334-8.

37. - Cardinale D, Ciceri F, Latini R, Franzosi MG, Sandri MT, Civelli $\mathrm{M}$, et al. Anthracycline-induced cardiotoxicity: a multicenter randomised trial comparing two strategies for guiding prevention with enalapril: the International CardioOncology Society-one trial. Eur J Cancer. 2018;94:126-37. Study showing the benefits of enalapril in patients with CTIC.

38. - Gulati G, Heck SL, Ree AH, Hoffmann P, Schulz-Menger J, Fagerland MW, et al. Prevention of cardiac dysfunction during adjuvant breast cancer therapy (PRADA): a $2 \times 2$ factorial, randomized, placebo-controlled, double-blind clinical trial of candesartan and metoprolol. Eur Heart J. 2016;37:1671-80. Study showing a benefit of candesartan in patients with CTIC.

39. - Avila MS, Ayub-Ferreira SM, de Barros Wanderley MR, das Dores Cruz F, Gonçalves Brandão SM, Rigaud VOC, et al. Carvedilol for prevention of chemotherapy-related cardiotoxicity: the CECCY trial. J Am Coll Cardiol. 2018;71:2281-90. Study showing the benefits of carvedilol in patients with CTIC.

40. Lenihan DJ, Stevens PL, Massey M, Plana JC, Araujo DM, Fanale MA, et al. The utility of point-of-care biomarkers to detect cardiotoxicity during anthracycline chemotherapy: a feasibility study. J Card Fail Elsevier Inc. 2016;22:433-8.

41. Palumbo I, Palumbo B, Fravolini ML, Marcantonini M, Perrucci E, Latini ME, et al. Brain natriuretic peptide as a cardiac marker of transient radiotherapy-related damage in left-sided breast cancer patients: a prospective study. Breast. 2016;25:45-50.

42. Bouwer NI, Liesting C, Kofflard MJM, Sprangers-van Campen SM, Brugts JJ, Kitzen JJEM, et al. NT-proBNP correlates with LVEF decline in HER2-positive breast cancer patients treated with trastuzumab. Cardio-Oncol. 2019;5:1-10.

43. Demissei B, Hubbard RA, Zhang L, Smith AM, Sheline K, McDonald C, et al. Changes in cardiovascular biomarkers with breast cancer therapy and associations with cardiac dysfunction. J Am Coll Cardiol. 2019;73:678.

44. Alvi RM, Frigault MJ, Fradley MG, Jain MD, Mahmood SS, Awadalla M, et al. Cardiovascular events among adults treated with chimeric antigen receptor T-cells (CAR-T). J Am Coll Cardiol. 2019;74:3099-108.

45. Cameron D, Piccart-Gebhart MJ, Gelber RD, Procter M, Goldhirsch A, de Azambuja E, et al. 11 years' follow-up of trastuzumab after adjuvant chemotherapy in HER2-positive early breast cancer: final analysis of the HERceptin Adjuvant (HERA) trial. Lancet Elsevier Ltd. 2017;389:1195-205.

46. Mohan N, Jiang J, Dokmanovic M, Wu WJ. Trastuzumab-mediated cardiotoxicity: current understanding, challenges, and frontiers. Antib Ther. 2018;1:13-7.

47. Mahmood SS, Fradley MG, Cohen JV, Nohria A, Reynolds KL, Heinzerling LM, et al. Myocarditis in patients treated with immune checkpoint inhibitors. J Am Coll Cardiol. 2018;71:1755-64.

48. Moslehi JJ, Salem JE, Sosman JA, Lebrun-Vignes B, Johnson DB. Increased reporting of fatal immune checkpoint inhibitor-associated myocarditis. Lancet. 2018:933.

49. Upadhrasta S, Elias H, Patel K, Zheng L. Managing cardiotoxicity associated with immune checkpoint inhibitors. Chronic dis Transl med. Elsevier Masson SAS. 2019;5:6-14.

50. Salem JE, Manouchehri A, Moey M, Lebrun-Vignes B, Bastarache L, Pariente A, et al. Cardiovascular toxicities associated with immune checkpoint inhibitors: an observational, retrospective, pharmacovigilance study. Lancet Oncol. 2018;19:1579-89.

51. Dolladille C, Ederhy S, Allouche S, Dupas Q, Gervais R, Madelaine $\mathrm{J}$, et al. Late cardiac adverse events in patients with cancer treated with immune checkpoint inhibitors. J Immunother cancer. 2020;8:e00261.

52. Lancellotti P, Moonen M, Galderisi M. Chimeric antigen receptor T-cells and cardiovascular toxicity: cause for concern? J Am Coll Cardiol. 2019:3109-11.

53. Pareek N, Cevallos J, Moliner P, Shah M, Tan LL, Chambers V, et al. Activity and outcomes of a cardio-oncology service in the United Kingdom - a five-year experience. Eur J Heart Fail. 2018;20:1721-31

54. Kappel C, Rushton M, Johnson C, Aseyev O, Small G, Law AJ, et al. Clinical experience of patients referred to a multidisciplinary cardio-oncology clinic: an observational cohort study. Curr Oncol. 2019;26:322-7.

55. Jaffe AS, Ordonez-Llanos J. High-sensitivity cardiac troponin: from theory to clinical practice. Rev Esp Cardiol. 2013.

56. Lipshultz SE, Rifai N, Sallan SE, Lipsitz SR, Dalton V, Sacks DB, et al. Predictive value of cardiac troponin $\mathrm{T}$ in pediatric patients at risk for myocardial injury. Circulation. 1997;96:2641-8.

57. Cardinale D, Sandri MT, Martinoni A, Tricca A, Civelli M, Lamantia $\mathrm{G}$, et al. Left ventricular dysfunction predicted by early troponin I release after high-dose chemotherapy. J Am Coll Cardiol. 2000;36:517-22.

58. Cardinale D, Sandri MT, Colombo A, Colombo N, Boeri M, Lamantia G, et al. Prognostic value of troponin I in cardiac risk 
stratification of cancer patients undergoing high-dose chemotherapy. Circulation. 2004;109:2749-54.

59. Rocco E, La Rosa G, Liuzzo G, Biasucci LM. High-sensitivity cardiac troponin assays and acute coronary syndrome: a matter of sex? J Cardiovasc Med. 2019:504-9.

60. Goel S, Liu J, Guo H, Barry W, Bell R, Murray B, et al. Decline in left ventricular ejection fraction following anthracyclines predicts trastuzumab cardiotoxicity. JACC Hear Fail. 2019;7:795-804.

61. Cadeddu Dessalvi C, Pepe A, Penna C, Gimelli A, Madonna R, Mele D, et al. Sex differences in anthracycline-induced cardiotoxicity: the benefits of estrogens. Heart Fail Rev. 2019: 915-25.

62. Kurz K, Giannitsis E, Zehelein J, Katus HA. Highly sensitive cardiac troponin $\mathrm{T}$ values remain constant after brief exercise- or pharmacologic-induced reversible myocardial ischemia. Clin Chem. 2008;54:1234-8.

63. Samaha E, Avila A, Helwani MA, Ben Abdallah A, Jaffe AS, Scott MG, et al. High-sensitivity cardiac troponin after cardiac stress test: a systematic review and meta-analysis. J Am Heart Assoc. 2019;8: e008626.

64. Ponikowski P, Voors AA, Anker SD, Bueno H, Cleland JGF, Coats AJS, et al. ESC guidelines for the diagnosis and treatment of acute and chronic heart failure. Eur Heart J. 2016, 2016:2129-2200m.

65. Mueller C, McDonald K, de Boer RA, Maisel A, Cleland JGF, Kozhuharov N, et al. Heart Failure Association of the European Society of Cardiology practical guidance on the use of natriuretic peptide concentrations. Eur J Heart Fail. 2019;21:715-31.

66. Wolsk E, Claggett B, Pfeffer MA, Diaz R, Dickstein K, Gerstein $\mathrm{HC}$, et al. Role of B-type natriuretic peptide and N-terminal prohormone BNP as predictors of cardiovascular morbidity and mortality in patients with a recent coronary event and type 2 diabetes mellitus. J Am Heart Assoc. 2017;6.
67. Suthahar N, Meijers WC, Ho JE, Gansevoort RT, Voors AA, van der Meer P, et al. Sex-specific associations of obesity and Nterminal pro-B-type natriuretic peptide levels in the general population. Eur J Heart Fail. 2018;20:1205-14.

68. DErrico MP, Petruzzelli MF, Gianicolo EAL, Grimaldi L, Loliva F, Tramacere F, et al. Kinetics of B-type natriuretic peptide plasma levels in patients with left-sided breast cancer treated with radiation therapy: results after one-year follow-up. Int J Radiat Biol. 2015;91: 804-9.

69. Ponde N, Bradbury I, Lambertini M, Ewer M, Campbell C, Ameels $\mathrm{H}$, et al. Cardiac biomarkers for early detection and prediction of trastuzumab and/or lapatinib-induced cardiotoxicity in patients with HER2-positive early-stage breast cancer: a NeoALTTO sub-study (BIG 1-06). Breast Cancer Res Treat. 2018;168:631-8.

70. De Iuliis F, Salerno G, Taglieri L, Lanza R, Cardelli P, Scarpa S. Circulating neuregulin-1 and galectin-3 can be prognostic markers in breast cancer. Int J Biol Markers. 2017;32:e333-6.

71. Dessì M, Madeddu C, Piras A, Cadeddu C, Antoni G, Mercuro G, et al. Long-term, up to 18 months, protective effects of the angiotensin II receptor blocker telmisartan on Epirubin-induced inflammation and oxidative stress assessed by serial strain rate. Springerplus. 2013;2:1-10.

72. Riddell E, Lenihan D. The role of cardiac biomarkers in cardiooncology. Curr Probl Cancer. 2018:375-85.

73. Strimbu K, Tavel JA. What are biomarkers? Curr Opin HIV AIDS. 2010:463-6.

Publisher's Note Springer Nature remains neutral with regard to jurisdictional claims in published maps and institutional affiliations. 